\title{
Supporting Information for: \\ Simultaneous Determination of Spectral Signatures and Decay Kinetics of Excited State Species in Semiconductor Nanocrystals Probed by Transient Absorption Spectroscopy
}

Tais Labrador', Gordana Dukovic*

*Department of Chemistry, University of Colorado, Boulder, Colorado 80309, United States

†School of Science, Saint Thomas University, Florida, 33054

*Corresponding author; E-mail: Gordana.Dukovic@colorado.edu

I. RAW TRANSIENT ABSORPTION (TA) SPECTRA:
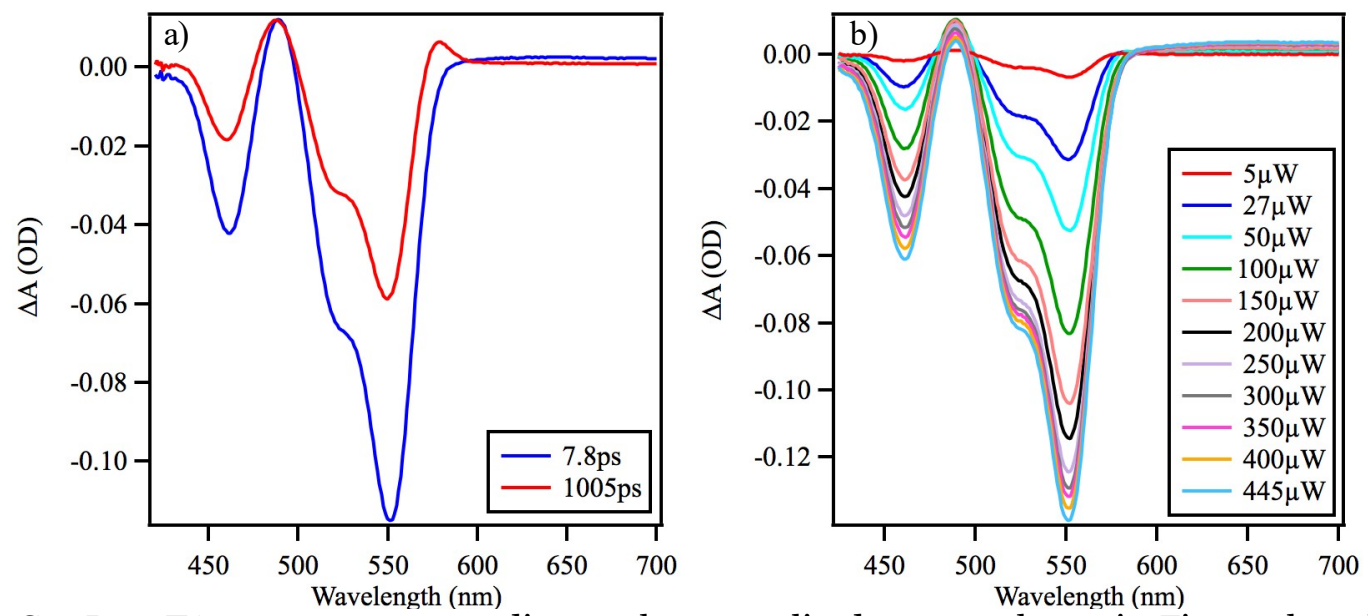

Figure S1: Raw TA spectra corresponding to the normalized spectra shown in Figure $1 \mathrm{~b}$ and $\mathrm{c}$ of the manuscript text. a) TA spectra collected at two different time delays at an excitation power of $250 \mu \mathrm{W} b$ ) TA spectra collected at different excitation powers at a single time delay (4.4 ps).

II. DERIVATION OF FIT EQUATIONS AND ANALYSIS RESULTS

A. EXTRACTING THE BIEXCITON SPECTRUM: DERIVATION OF EQUATION 2 OF THE MAIN TEXT AND ANALYSIS RESULTS IN THE 3-500 PS TIME WINDOW

During a TA experiment the $\Delta \mathrm{A}$ spectrum collected at any given time contains contributions from sample absorption and stimulated emission'1. In the derivation presented below, we ignore stimulated emission. We believe that this assumption is reasonable, as others have found that CdSe QDs photoluminescence is affected by post-synthetic purification procedures, resulting in QDs with low photoluminescence quantum yields ${ }^{2-3}$. As we only consider the contribution of sample absorption, we use the standard sign convention and define the $\Delta \mathrm{A}$ spectrum observed at any given time in a TA experiment $\Delta A(\lambda, t)$ as the difference between the absorption spectrum obtained when the sample interacts with the pump beam (i.e. the "pump on" absorption spectrum, $A(\lambda, t)_{p-o n}$ ) and that obtained when the sample does not interact with the pump beam, (i.e. the "pump-off" absorption spectrum, $A(\lambda, t)_{p-o f f}$ ):

$$
\Delta A(\lambda, t)=A(\lambda, t)_{p-o n}-A(\lambda, t)_{p-o f f}
$$

For a sample with multiple possible excited state populations:

$$
\Delta A(\lambda, t)=\left[\sum_{n=1}^{q} p_{n}(t) \Delta A_{n}(\lambda)\right]
$$


where $q$ is the total number of excited state populations generated through interaction with the pump beam, $p_{n}(t)$ is the time dependent probability of finding the sample in the n-excited state, and $\Delta A_{n}(\lambda)$ is the $\Delta \mathrm{A}$ spectrum corresponding to the n-excited state population.

The experiments presented here are performed on an ensemble of QDs. Interaction with the pump beam generates an initial distribution of n-exciton populations. These populations decay through a series of sequential steps in which every n-exciton population decays into the n-1 population, and the series continues until all excited nanocrystals have a single exciton, at which point, they all decay into the ground state4. The recombination dynamics of such set of sequential processes is typically described through a set of coupled differential equations ${ }^{4-5}$, whose solution simplifies to equation $\mathrm{S}^{6}{ }^{6}$ under the assumption that $\tau_{n+1}<<\tau_{n}$.

$$
p_{n}(t)=\sum_{i=n}^{\infty} p_{i} e^{-t / \tau_{n}}-\sum_{i=n+1}^{\infty} p_{i} e^{-t / \tau_{n+1}}
$$

Where $p_{i}$ is the probability of finding the nanocrystal in a given exciton state at time zero, $p_{n}(t)$ is the time dependent probability of finding the nanocrystal in the n-exciton state, and $\tau_{n}$ and $\tau_{n+1}$ are the time constants corresponding to the decay of the $\mathrm{n}$ and $\mathrm{n}+1$ exciton states.

We substitute Equation S3 into Equation S2 to obtain Equation 2 of the main text (labeled as Equation S4 for consistency here) and test through analysis whether the resultant equation applies to the biexciton and single exciton recombination processes occurring on the data shown in Figure S1:

$$
\Delta A(\lambda, t)=\sum_{n=1}^{q} \Delta A(\lambda)_{n}\left[\sum_{i=n}^{\infty} p_{i} e^{-t / \tau_{n}}-\sum_{i=n+1}^{\infty} p_{i} e^{-t / \tau_{n+1}}\right]
$$

Equation $\mathrm{S} 4$ can be rearranged into a more convenient fit equation (Equation 2a in the manuscript text, labeled S4a here for consistency), where the exponential corresponding to the recombination of the nexciton state is multiplied by a spectral signal $S_{n}(\lambda)$. The relationship between the $S_{n}(\lambda)$ and the spectra of the $\mathrm{n}$-exciton states is given by Equations $\mathrm{S} 4 \mathrm{~b}$ and $\mathrm{S} 4 \mathrm{c}$ (labeled $2 \mathrm{~b}$ and $\mathrm{c}$ in the manuscript text).

$$
\begin{aligned}
& \Delta A(\lambda, t)=\sum_{n=1}^{q} S_{n}(\lambda) e^{-\frac{t}{\tau_{n}}} \\
& S_{1}(\lambda)=\sum_{i=1}^{\infty} p_{i} \Delta A_{1}(\lambda) \\
& S_{n}(\lambda)=\sum_{i=n}^{\infty} p_{i}\left[\Delta A_{n}(\lambda)-\Delta A_{n-1}(\lambda)\right]
\end{aligned}
$$

Equation S4a was used to analyze the data sets extracted at all excitation powers. As a result, we extracted the pre-exponential coefficients that multiply the single exponential decay terms corresponding to the single exciton, biexciton, and triexciton states, respectively (See figures $2 \mathrm{a}$ and $2 \mathrm{~b}$ in the manuscript text for $S_{1}(\lambda)$ and $S_{2}(\lambda)$ ), and the time constants corresponding to the recombination of the single exciton, biexciton, and triexciton states (Table $\mathrm{S} 1$ ). Figure $\mathrm{S} 2 \mathrm{a}$ and b shows the $S_{1}(\lambda)$ and $S_{2}(\lambda)$ signals divided by the fraction of excited QDs and the fraction of excited QDs with multiexcitons, respectively. The greatest deviation is observed in the $S_{2}(\lambda)$ signal obtained at $100 \mu \mathrm{W}$. This signal deviates by $\sim 6 \%$ from the others at the position of the ground state bleach $(557 \mathrm{~nm})$.

\begin{tabular}{|c|c|c|c|}
\hline $\begin{array}{c}\text { Excitation } \\
\text { power } \\
(\mu \mathbf{W})\end{array}$ & $\begin{array}{c}\text { Single exciton } \\
\text { recombination } \\
\tau_{\mathbf{1}}(\mathbf{p s})\end{array}$ & $\begin{array}{c}\text { Biexciton } \\
\text { recombination } \\
\boldsymbol{\tau}_{\mathbf{2}} \mathbf{( p s )}\end{array}$ & $\begin{array}{c}\text { Triexciton } \\
\text { recombination } \\
\tau_{\mathbf{3}}(\mathbf{p s})\end{array}$ \\
\hline $\mathbf{5}$ & $2184 \pm 31$ & $39.38 \pm 1.57$ & $2.96 \pm 0.30$ \\
\hline $\mathbf{2 7}$ & $3084 \pm 24$ & $49.92 \pm 0.58$ & $6.08 \pm 0.10$ \\
\hline $\mathbf{5 0}$ & $2459 \pm 11$ & $43.87 \pm 0.31$ & $5.54 \pm 0.05$ \\
\hline $\mathbf{1 0 0}$ & $2599 \pm 10$ & $36.98 \pm 0.15$ & $5.00 \pm 0.03$ \\
\hline $\mathbf{1 5 0}$ & $2748 \pm 8$ & $33.91 \pm 0.09$ & $5.00 \pm 0.03$ \\
\hline $\mathbf{2 0 0}$ & $2754 \pm 12$ & $34.92 \pm 0.11$ & $4.76 \pm 0.03$ \\
\hline $\mathbf{2 5 0}$ & $3043 \pm 14$ & $34.43 \pm 0.11$ & $4.90 \pm 0.02$ \\
\hline $\mathbf{3 0 0}$ & $3063 \pm 17$ & $34.12 \pm 0.12$ & $4.57 \pm 0.02$ \\
\hline $\mathbf{3 5 0}$ & $3206 \pm 21$ & $34.61 \pm 0.13$ & $4.28 \pm 0.02$ \\
\hline $\mathbf{4 0 0}$ & $3385 \pm 26$ & $34.79 \pm 0.14$ & $4.20 \pm 0.02$ \\
\hline $\mathbf{4 4 5}$ & $3475 \pm 28$ & $34.60 \pm 0.13$ & $4.14 \pm 0.02$ \\
\hline
\end{tabular}

Table S1: Time constants obtained by fitting the data at different excitation powers in the time window between 3 and 500 ps. 

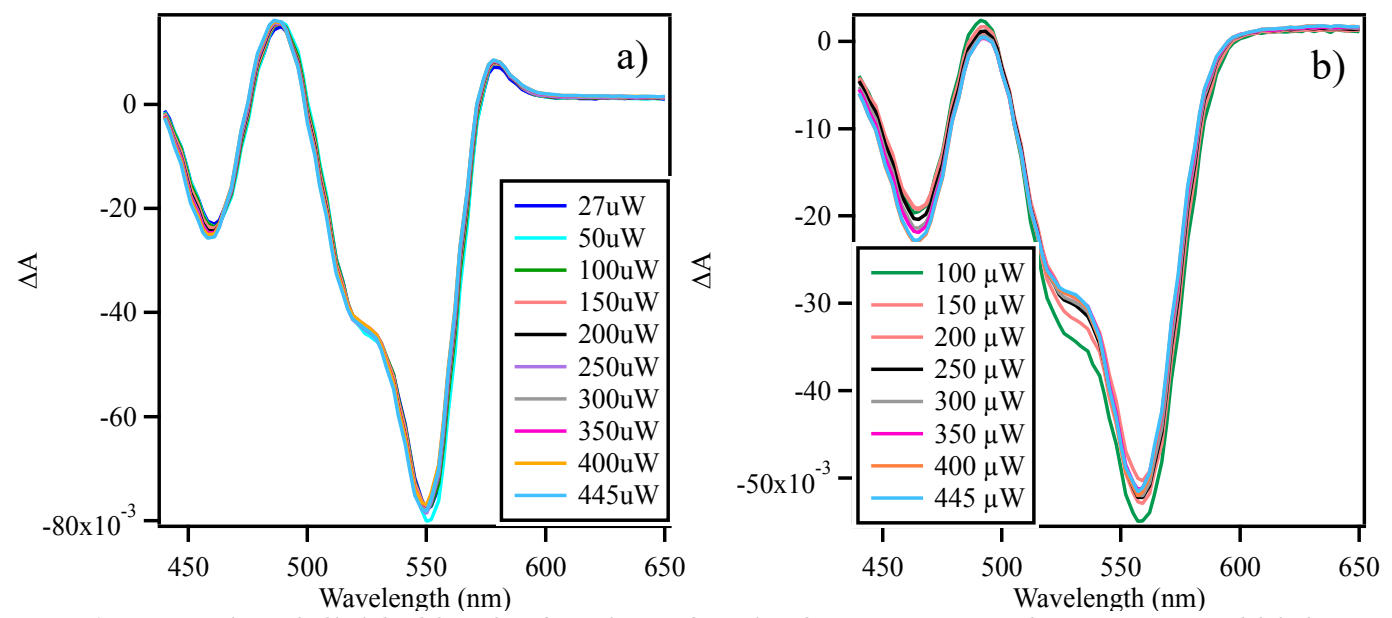

Figure S2: a) $S_{1}(\lambda)$ signal divided by the fraction of excited QDs extracted at $27 \mu \mathrm{W}$ and higher excitation powers. b) $S_{2}(\lambda)$ signal divided by the fraction of excited QDs with multiexcitons extracted at $100 \mu \mathrm{W}$ and higher excitation powers.

In the manuscript text we described how the spectral signals obtained through the analysis of the data obtained at all excitation powers can be used to determine the average number of excited QDs initially present in the ensemble along with the fractions of total excited QDs and total excited QDs with multiexcitons. The results are shown in Table S2:

\begin{tabular}{|r|c|c|c|}
\hline $\begin{array}{c}\text { Excitation } \\
\text { power }\end{array}$ & $<\mathbf{N}>$ & $\begin{array}{c}\text { Total fraction of } \\
\text { excited QDs } \\
\left(\sum_{i=1}^{\infty} \boldsymbol{p}_{\boldsymbol{i}}\right)\end{array}$ & $\begin{array}{c}\text { Fraction of } \\
\text { excited QDs with } \\
\text { multiexcitons } \\
\left(\sum_{i=2}^{\infty} \boldsymbol{p}_{\boldsymbol{i}}\right)\end{array}$ \\
\hline $\mathbf{5}$ & $0.073 \pm 0.001$ & $0.0699 \pm 0.0009$ & $0.00250 \pm 0.00007$ \\
\hline $\mathbf{2 7}$ & $0.393 \pm 0.005$ & $0.3240 \pm 0.0037$ & $0.0593 \pm 0.0014$ \\
\hline $\mathbf{5 0}$ & $0.728 \pm 0.010$ & $0.5157 \pm 0.0048$ & $0.1645 \pm 0.0035$ \\
\hline $\mathbf{1 5 0}$ & $2.183 \pm 0.030$ & $0.8864 \pm 0.0034$ & $0.6393 \pm 0.0074$ \\
\hline $\mathbf{2 0 0}$ & $2.910 \pm 0.040$ & $0.9450 \pm 0.0022$ & $0.7854 \pm 0.0064$ \\
\hline $\mathbf{2 5 0}$ & $3.638 \pm 0.050$ & $0.9734 \pm 0.0013$ & $0.8767 \pm 0.0048$ \\
\hline $\mathbf{3 0 0}$ & $4.365 \pm 0.060$ & $0.9871 \pm 0.0008$ & $0.9309 \pm 0.0034$ \\
\hline $\mathbf{3 5 0}$ & $5.093 \pm 0.070$ & $0.9937 \pm 0.0004$ & $0.9620 \pm 0.0022$ \\
\hline $\mathbf{4 0 0}$ & $5.820 \pm 0.080$ & $0.9970 \pm 0.0002$ & $0.9794 \pm 0.0014$ \\
\hline $\mathbf{4 4 5}$ & $6.475 \pm 0.089$ & $0.9984 \pm 0.0001$ & $0.9883 \pm 0.0009$ \\
\hline
\end{tabular}

Table S2: Average number of excited QDs and fractions of total excited QDs and total excited QDs with multiexcitons obtained at different excitation powers.

\section{B. EXTRACTING THE BIEXCITON SPECTRUM: DERIVATION OF EQUATION SUITABLE FOR ANALYSIS IN THE 3 ps-3 ns TIME WINDOW AND RESULTS OF ANALYSIS}

In deriving Equation $\mathrm{S} 4$, we assumed that the decay of all n-exciton states can be described using single exponential functions. We have found that this assumption is appropriate in all cases presented here for the multiexciton states, while for the single exciton state, the assumption is only valid in the time window extending from 3 ps to 500 ps. Analysis of the data sets collected at all excitation powers to the end of the experimental time window, at $3 \mathrm{~ns}$, requires a biexponential function to describe the decay of the single 
exciton state. Therefore, to perform analysis in the time window of 3 ps to $3 \mathrm{~ns}$, it is necessary to obtain an expression that describes the dynamics of the single exciton state taking into account the multiexponential nature of its decay.

The functional form of a recombination process with an arbitrary decay kinetics can be obtained considering that such process has a time-dependent rate constant.7 To describe the kinetics of the single exciton state, we start with the following expression:

$$
\frac{d p_{1}(t)}{d t}=\frac{p_{2}(t)}{\tau_{2}}-k_{1}(t) p_{1}(t)
$$

where $p_{1}(t)$ and $p_{2}(t)$ are the time dependent probabilities of finding the nanocrystal in the single exciton and biexciton states, $\tau_{2}$ and $k_{1}(t)$ are the time constant and time dependent rate constant corresponding to the decay of biexciton and single exciton states, respectively.

The general solution to Equation $\mathrm{S}_{5}$ is:

$$
p_{1}(t)=I(t) \int_{0}^{t} \frac{p_{2}(z)}{I(z)} d z+I(t) \frac{p_{1}(0)}{I(0)},
$$

where $I(t)$ corresponds to the functional form of the decay of the single exciton state. For the specific case in which $I(t)$ is a biexponential function of the form $I(t)=C e^{-t / \tau_{a}}+(1-C) e^{-t / \tau_{b}}$, and assuming that $\tau_{a}>>\tau_{2}$ and $\tau_{b}>>\tau_{2}$, the solution to Equation S6 converges to:

$$
p_{1}(t)=\sum_{i=1}^{\infty} p_{i}\left[C e^{-t / \tau_{a}}+(1-C) e^{-t / \tau_{b}}\right]-\sum_{i=2}^{\infty} p_{i} e^{-t / \tau_{2}}
$$

Where $\tau_{a}$ and $\tau_{b}$ are the time constants corresponding to the decay of the single exciton state and $C$ is a constant parameter whose value determines the pre-exponential coefficients of $I(t)$.

To obtain a modified $\Delta A(\lambda, t)$ expression that takes into account the multiexponential nature of the decay dynamics of the single exciton state, we start with the general form of $\Delta A(\lambda, t)$ given by Equation S2. The functional forms of the time dependent probability of finding the QDs in the single exciton state is given by Equation S7, while that of the multiple exciton states are given by Equation $\mathrm{S}_{3}$. Therefore, we substitute Equations $\mathrm{S} 3$ and $\mathrm{S} 7$ into Equation $\mathrm{S} 2$ to obtain the modified $\Delta A(\lambda, t)$ expression:

$$
\begin{aligned}
\Delta A(\lambda, t)= & \Delta A(\lambda)_{1}\left[\sum_{i=1}^{\infty} p_{i}\left[C e^{-t / \tau_{a}}+(1-C) e^{-t / \tau_{b}}\right]-\sum_{i=2}^{\infty} p_{i} e^{-t / \tau_{2}}\right]+ \\
& +\sum_{n=2}^{q} \Delta A(\lambda)_{n}\left[\sum_{i=n}^{\infty} p_{i} e^{-t / \tau_{n}}-\sum_{i=n+1}^{\infty} p_{i} e^{-t / \tau_{n+1}}\right]
\end{aligned}
$$

Equation S8 can be rearranged into Equation S8a, which is a more convenient fit equation, as it describes the data set as a sum of a biexponential function with a wavelength dependent pre-exponential coefficient, $S_{1}(\lambda)$, and single exponential functions with wavelength dependent pre-exponential coefficients, $S_{n}(\lambda)$ for multi-excitons.

$$
\Delta A(\lambda, t)=S_{1}(\lambda)\left[C e^{-t / \tau_{a}}+(1-C) e^{-t / \tau_{b}}\right]+\sum_{n=2}^{q} S_{n}(\lambda) e^{-\frac{t}{\tau_{n}}}
$$

where:

$S_{1}(\lambda)=\sum_{i=1}^{\infty} p_{i} \Delta A_{1}(\lambda)$

$S_{n}(\lambda)=\sum_{i=n}^{\infty} p_{i}\left[\Delta A_{n}(\lambda)-\Delta A_{n-1}(\lambda)\right]$

We randomly selected data sets collected at several excitation powers and analyzed each one individually using Equation S8a in the time window of 3 ps to $3 \mathrm{~ns}$. In all cases, a biexponential function with $\tau_{a}$ and $\tau_{b}$ time constants, a constant parameter $C$ and a set of $S_{1}(\lambda)$ pre-exponential coefficients was needed to describe the decay of the single exciton state. A sum of two exponentials with $\tau_{2}$ and $\tau_{3}$ time constants and two sets of pre-exponential coefficients, $S_{2}(\lambda)$ and $S_{3}(\lambda)$, were sufficient to describe the decay of the biexciton and triexciton states. All time constants and the constant parameter $C$ were treated as global parameters, while the three sets of pre-exponential coefficients, $S_{1}(\lambda), S_{2}(\lambda)$, and $S_{3}(\lambda)$ were allowed to vary for every kinetic trace analyzed.

These fits yielded four time constants, $\tau_{a}, \tau_{b}, \tau_{2}$, and $\tau_{3}$, a constant parameter $C$, and three sets of pre-exponential coefficients $S_{1}(\lambda), S_{2}(\lambda)$, and $S_{3}(\lambda)$. Table $S_{3}$ shows the time constants obtained at all excitation powers chosen for analysis. Figure $S_{3}$ a and b show the $S_{1}(\lambda)$, and $S_{2}(\lambda)$ pre-exponential coefficients obtained through this analysis. Our goal is to evaluate whether the same $S_{1}(\lambda)$, and $S_{2}(\lambda)$ preexponential coefficients and time constants are obtained regardless of the time window and fit function chosen for analysis. Note that similar $\tau_{2}$ and $\tau_{3}$ time constants are reported in Tables S3 and S1. The $S_{1}(\lambda)$ and $S_{2}(\lambda)$ pre-exponential coefficients shown in Figure $S_{3}$ overlap at each excitation powers for both time windows with minor deviations. 


\begin{tabular}{|c|c|c|c|c|}
\hline $\begin{array}{c}\text { Excitation } \\
\text { power } \\
(\boldsymbol{\mu W})\end{array}$ & $\begin{array}{c}\text { Single exciton } \\
\text { recombination } \\
\tau_{\mathbf{a}}(\mathbf{p s})\end{array}$ & $\begin{array}{c}\text { Single exciton } \\
\text { recombination } \\
\tau_{\mathbf{b}} \mathbf{( p s )}\end{array}$ & $\begin{array}{c}\text { Biexciton } \\
\text { recombination } \\
\boldsymbol{\tau}_{\mathbf{2}} \mathbf{( p s )}\end{array}$ & $\begin{array}{c}\text { Triexciton } \\
\text { recombination } \\
\tau_{\mathbf{3}} \mathbf{( p s )}\end{array}$ \\
\hline $\mathbf{2 7}$ & $9441 \pm 58$ & $598 \pm 7$ & $49.47 \pm 0.52$ & $6.20 \pm 0.09$ \\
\hline $\mathbf{5 0}$ & $8104 \pm 23$ & $482 \pm 3$ & $43.93 \pm 0.27$ & $5.70 \pm 0.05$ \\
\hline $\mathbf{1 0 0}$ & $8645 \pm 24$ & $526 \pm 3$ & $37.37 \pm 0.12$ & $5.16 \pm 0.02$ \\
\hline $\mathbf{1 5 0}$ & $8865 \pm 22$ & $518 \pm 3$ & $34.42 \pm 0.09$ & $5.30 \pm 0.02$ \\
\hline $\mathbf{2 0 0}$ & $8821 \pm 21$ & $470 \pm 3$ & $35.28 \pm 0.09$ & $5.00 \pm 0.02$ \\
\hline $\mathbf{2 5 0}$ & $9825 \pm 35$ & $576 \pm 4$ & $35.13 \pm 0.08$ & $5.11 \pm 0.02$ \\
\hline $\mathbf{3 5 0}$ & $10174 \pm 48$ & $577 \pm 6$ & $35.43 \pm 0.10$ & $4.47 \pm 0.02$ \\
\hline
\end{tabular}

Table S3: Time constants obtained by fitting the TA data to Equation S8a at different excitation powers in the time window between 3 ps and 3 ns.
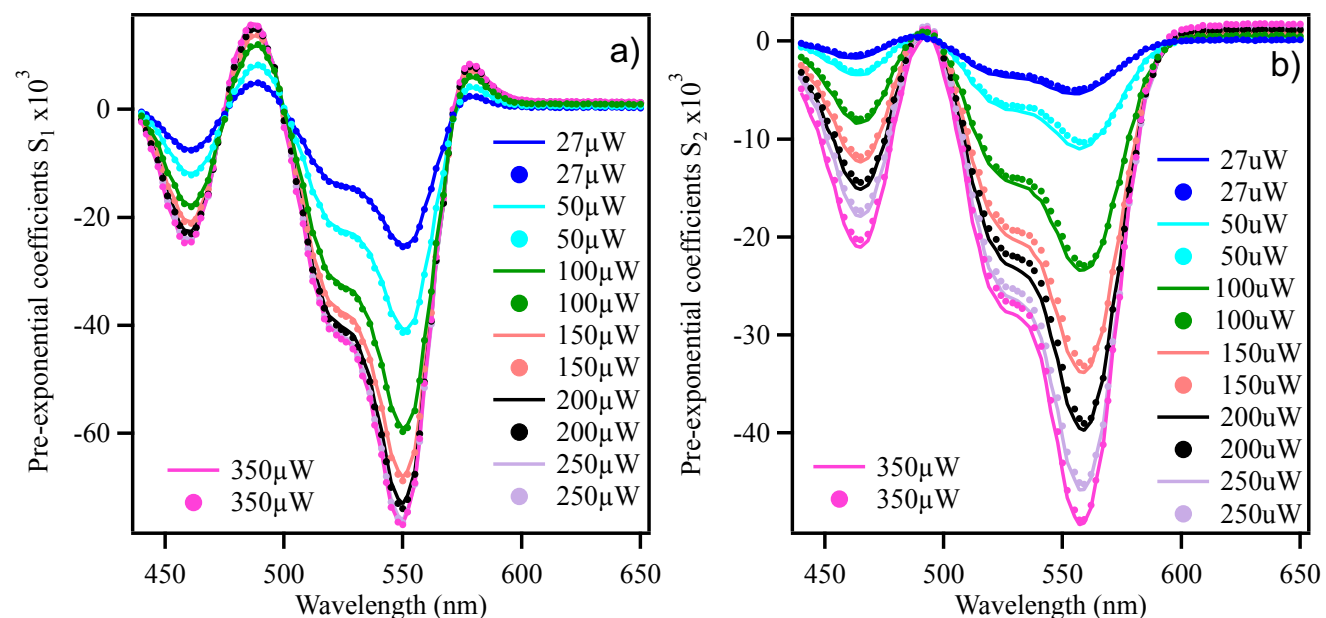

Figure S3: Comparison of the pre-exponential coefficients (a) $S_{1}(\lambda)$ and (b) $S_{2}(\lambda)$ extracted through analysis in the 3 ps to 500 ps (solid lines) and 3 ps to 3 ns time windows (dotted lines).

III. BIEXCITON TA SPECTRA EXTRACTED AT $100 \mu \mathrm{W}$ AND HIGHER PUMP POWERS.

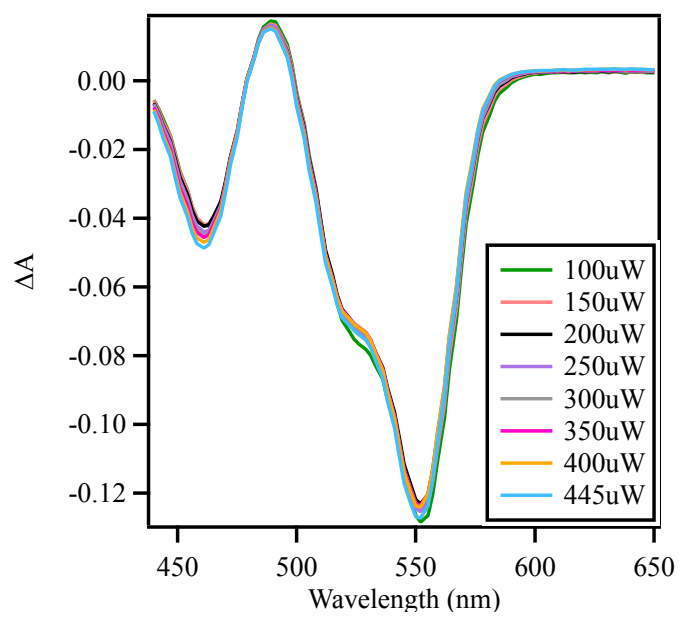

Figure S4: Biexciton TA spectra $\Delta A_{2}(\lambda)$ extracted at $100 \mu \mathrm{W}$ and higher excitation powers using Equation 5 of the main text. 


\section{MEASURING THE BIEXCITON AND TRIEXCITON BINDING ENERGIES}

To obtain the value of the biexciton and triexciton binding energy, we have fit the single exciton $\left(\Delta \mathrm{A}_{1}\right)$ and biexciton spectra $\left(\Delta \mathrm{A}_{2}\right)$ simultaneously using a global fit routine and a linear combination of gaussians functions as fit equations (Equations $\mathrm{S} 12$ and $\mathrm{S} 13$ ) to describe the spectral line shapes of the $1 \mathrm{~S}_{\mathrm{e}^{-}}$ $1 \mathrm{~S}_{3 / 2(\mathrm{~h}),} 1 \mathrm{~S}_{\mathrm{e}}-2 \mathrm{~S}_{3 / 2(\mathrm{~h})}, 1 \mathrm{~S}_{\mathrm{e}}-2 \mathrm{~S}_{1 / 2(\mathrm{~h})}$, and $1 \mathrm{P}_{\mathrm{e}}-1 \mathrm{P}_{3 / 2(\mathrm{~h})}$ of the ground and excited state, following the methods reported in the past to extract the biexciton binding energies from TA spectra of CdSe QDs ${ }^{8-9}$. In equations S12 and S13, the transitions are labeled using the labeling scheme for the hole energy level.

$$
\begin{array}{r}
\Delta \mathrm{A}_{1}=A\left[( \frac { 1 } { 2 } e ^ { - \frac { ( E - c _ { 1 S _ { 3 } } - \Delta _ { x x } ) ^ { 2 } } { 2 w ^ { 2 } } } - e ^ { - \frac { ( E - c _ { 1 S _ { 3 } } ) ^ { 2 } } { 2 w ^ { 2 } } ) } + f _ { 2 S _ { \frac { 3 } { 2 } } } ( \frac { 1 } { 2 } e ^ { - \frac { ( E - c _ { 2 S _ { 3 } } - \Delta _ { x x } } { 2 w ^ { 2 } } } - e ^ { - \frac { ( E - c _ { 2 S _ { 3 } } ) ^ { 2 } } { 2 w ^ { 2 } } ) } ) ^ { 2 } ) ^ { 2 } \left(f_{1 P_{\frac{3}{2}}}\left(e^{-\frac{\left(E-c_{1 P_{3}}-\Delta_{x x}\right)^{2}}{2 w^{2}}}-e^{-\frac{\left(E-c_{1 P_{3}}\right)^{2}}{2 w^{2}}}\right)+f_{2 S_{\frac{1}{2}}}\left(\frac{1}{2} e^{-\frac{\left(E-c_{2 S_{1}}-\Delta_{x x}\right)^{2}}{2 w^{2}}}-e^{\left.\left.-\frac{\left(E-c_{2 S_{1}}\right)^{2}}{2 w^{2}}\right)\right]}\right.\right.\right. \\
\Delta \mathrm{A}_{2}=A\left[-e^{-\frac{\left(E-c_{1 S_{3 / 2}}\right)^{2}}{2 w^{2}}}-f_{2 S_{3 / 2}} e^{-\frac{\left(E-c_{2 S_{3 / 2}}\right)^{2}}{2 w^{2}}}-f_{2 S_{1 / 2}} e^{-\frac{\left(E-c_{2 S_{1 / 2}}\right)^{2}}{2 w^{2}}}+f_{1 P_{3 / 2}}\left(e^{-\frac{\left(E-c_{1 P_{3 / 2}}-\Delta_{x x x}\right)^{2}}{2 w^{2}}}-e^{\left.-\frac{\left(E-c_{1 P_{3 / 2}}\right)^{2}}{2 w^{2}}\right)}\right)\right]
\end{array}
$$

$\mathrm{S} 12$

where $c$ represent the centers of the peaks associated with the $1 \mathrm{~S}_{\mathrm{e}}-1 \mathrm{~S}_{3 / 2(\mathrm{~h})},{ }_{1} \mathrm{~S}_{\mathrm{e}}-2 \mathrm{~S}_{3 / 2(\mathrm{~h})}, 1 \mathrm{~S}_{\mathrm{e}}-2 \mathrm{~S}_{1 / 2(\mathrm{~h})}$, and $1 \mathrm{P}_{\mathrm{e}}-$ ${ }_{1} \mathrm{P}_{3 / 2(\mathrm{~h})}$ transitions (labeled in the equations by the hole state), and $w$ represents the width of those peaks, $\Delta_{x x}$ and $\Delta_{x x x}$ are the biexciton and triexciton binding energies, respectively, $A$ is the amplitude of the TA spectra, while the $f_{2 S_{3 / 2}}, f_{2 S_{1 / 2}}$, and $f_{1 P_{3 / 2}}$ coefficients are the ratios of the amplitudes of the peaks associated with the $1 \mathrm{~S}_{\mathrm{e}}-2 \mathrm{~S}_{3 / 2(\mathrm{~h})}, 1 \mathrm{~S}_{\mathrm{e}}-2 \mathrm{~S}_{1 / 2(\mathrm{~h})}$, and $1 \mathrm{P}_{\mathrm{e}}-1 \mathrm{P}_{3 / 2(\mathrm{~h})}$ transitions to that associated with the $1 \mathrm{~S}_{\mathrm{e}}-1 \mathrm{~S}_{3 / 2(\mathrm{~h})}$ transition.

For these fits, the centers $c$ and widths $w$ of all peaks, as well as the $f_{2 S_{3 / 2}}, f_{2 S_{1 / 2}}$, and $f_{1 P_{3 / 2}}$ factors were treated as global parameters. The width of all absorption peaks was assumed to be the same for all transitions. This analysis yielded values for the center, width, and amplitudes of all peaks, as well as the biexciton and triexciton binding energies (Table S4). Figure $\mathrm{S}_{5}$ shows the fits to the single exciton and biexciton spectra along with the residual traces.

\begin{tabular}{|c|c|c|}
\hline $\begin{array}{c}\text { Fitting } \\
\text { parameters }\end{array}$ & $\begin{array}{c}\text { Fitting results } \\
\text { (Single exciton } \\
\text { Spectrum) }\end{array}$ & $\begin{array}{c}\text { Fitting results } \\
\text { (Biexciton spectrum) }\end{array}$ \\
\hline $\boldsymbol{A}$ & $0.1186 \pm 0.0016$ & $0.0789 \pm 0.0008$ \\
\hline $\boldsymbol{f}_{2 s_{3 / 2}}$ & $0.5328 \pm 0.0104$ & $0.5328 \pm 0.0104$ \\
\hline $\boldsymbol{f}_{1 P_{3 / 2}}$ & $0.3631 \pm 0.0243$ & $0.3631 \pm 0.0243$ \\
\hline $\boldsymbol{f}_{2 s_{1 / 2}}$ & $0.2528 \pm 0.0101$ & $0.2528 \pm 0.0101$ \\
\hline $\boldsymbol{c}_{1 S_{3 / 2}}(\mathrm{eV})$ & $2.2437 \pm 0.0006$ & $2.2437 \pm 0.0006$ \\
\hline $\boldsymbol{c}_{2 S_{3 / 2}}(\mathbf{e V})$ & $2.3716 \pm 0.0012$ & $2.3716 \pm 0.0012$ \\
\hline $\boldsymbol{c}_{1 P_{3 / 2}}(\mathbf{e V})$ & $2.6115 \pm 0.0020$ & $2.6115 \pm 0.0020$ \\
\hline $\boldsymbol{c}_{2 S_{1 / 2}}(\mathbf{e V})$ & $2.7085 \pm 0.0025$ & $2.7085 \pm 0.0025$ \\
\hline $\boldsymbol{w}(\mathbf{e V})$ & $0.0511 \pm 0.0005$ & $0.0511 \pm 0.0005$ \\
\hline$\Delta_{x x}(\mathbf{e V})$ & $-0.0256 \pm 0.0013$ & -------------- \\
\hline$\Delta_{x x x}(\mathrm{eV})$ & ------------ & $-0.0351 \pm 0.0031$ \\
\hline
\end{tabular}

Table S4: Fitting results for the single exciton and biexciton spectra at $150 \mu \mathrm{W}$. 


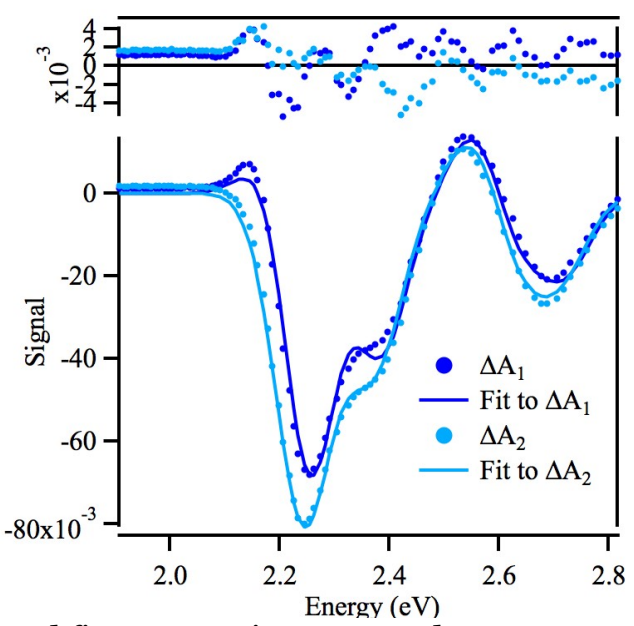

Figure S5: $\Delta A_{1}$ and $\Delta A_{2}$ spectra and fits to equations S12 and S13.

\section{EXTRACTING THE HOT EXCITON SPECTRUM: DERIVATION OF EQUATION SUITABLE FOR ANALYSIS IN THE 0-500 PS TIME WINDOW AND RESULTS OF ANALYSIS}

Equations S4 and S8 ignore the presence of hot exciton states, and therefore, they can only be employed in the analysis of the TA data collected after all hot exciton states have cooled to the band gap. In this section, we derive a $\Delta A(\lambda, t)$ expression that includes the kinetics and spectra of the hot excitons, with the purpose of utilizing it for the extraction of the spectrum corresponding to the hot exciton state. To accomplish this, we need an expression that encompasses the decay kinetics of both the hot and cold nexciton states. To obtain such an expression, we assume that upon interaction with the pump beam, every $\mathrm{n}$-exciton state in the nanocrystal ensemble is above the band gap. This is a reasonable assumption as the excitation wavelength used for the experiments presented here is lower than that of the bandgap ${ }^{8-11}$.

The hot n-excitons decay following a set of sequential steps, which start with their relaxation to the band gap. Once at the band gap, each n-exciton state decays into the n-1 state and the sequence of decay events ends when the only remaining state, the single exciton, decays into the ground state. Equation S9 and S10 model the dynamics of the hot and cold n-excitons considering that all $n$-exciton cooling and recombination processes can be modeled using single exponential functions $4-5$, 10-11, with $\tau_{n}^{c}$ and $\tau_{n}$ as the time constants corresponding to cooling and recombination of the $n$-exciton states, respectively. Equation S9 and S10 were derived under the assumption that $\tau_{n}^{c} \ll \tau_{n+1} \ll \tau_{n}$.

$$
\begin{gathered}
p_{n}^{h}(t)=p_{n} e^{-t / \tau_{n}^{c}} \\
p_{n}(t)=-p_{n} e^{-t / \tau_{n}^{c}}-\sum_{i=n+1}^{\infty} p_{i} e^{-t / \tau_{n+1}}+\sum_{i=n}^{\infty} p_{i} e^{-t / \tau_{n}}
\end{gathered}
$$

Where $p_{n}$ is the probability of finding the nanocrystal at a particular n-exciton state at time zero, $p_{n}(t)$ and $p_{n+1}(t)$ are the time dependent probabilities of the $\mathrm{n}$ and $\mathrm{n}+1$ exciton states at the band gap, respectively, $p_{n}^{h}(t)$ is the time dependent probability of finding the n-exciton state above the band gap (i.e., the " $\mathrm{h}$ " superscript stands for "hot"), $\tau_{n}$ and $\tau_{n+1}$ are the recombination time constants of the $\mathrm{n}$ and $\mathrm{n}+1$ exciton states, and $\tau_{n}^{c}$ is the cooling time constant of the n-exciton state (i.e., the "c" superscript stands for "cooled").

To obtain the desired $\Delta A(\lambda, t)$ expression, we substitute Equations S9 and S10 into Equation S2 and we consider that each hot and cold $\mathrm{n}$-exciton state generates a distinct spectrum:

$$
\Delta A(\lambda, t)=\sum_{n=1}^{q} \Delta A_{n}(\lambda)\left[-p_{n} e^{-t / \tau_{n}^{c}}-\sum_{i=n+1}^{\infty} p_{i} e^{-t / \tau_{n+1}}+\sum_{i=n}^{\infty} p_{i} e^{-t / \tau_{n}}\right]+\sum_{n=1}^{q} \Delta A_{n}^{h}(\lambda) p_{n} e^{-t / \tau_{n}^{c}} \quad \mathrm{~S} 11
$$

where $\Delta A_{n}(\lambda)$ are the spectra corresponding to the n-exciton state at the band gap, while $\Delta A_{n}^{h}(\lambda)$ are the spectra corresponding to the n-exciton state residing above the band gap.

Rearranging Equation S11, we obtain Equation S11a, which is a more convenient fit expression, as it describes a TA data set as a sum of exponentials with wavelength-dependent pre-exponential coefficients.

$$
\Delta A(\lambda, t)=\sum_{n=1}^{q} S_{n}(\lambda) e^{-t / \tau_{n}}+\sum_{n=1}^{q} S_{n}^{h}(\lambda) e^{-t / \tau_{n}^{c}}
$$

where the $S_{n}(\lambda)$ and $S_{n}^{h}(\lambda)$ pre-exponential coefficients are given by:

$$
S_{1}(\lambda)=\sum_{i=1}^{\infty} p_{i} \Delta A_{1}(\lambda)
$$




$$
\begin{array}{cc}
S_{n}(\lambda)=\sum_{i=n}^{\infty} p_{i}\left[\Delta A_{n}(\lambda)-\Delta A_{n-1}(\lambda)\right] & \text { S11c } \\
S_{n}^{h}(\lambda)=p_{n}\left[\Delta A_{n}^{h}(\lambda)-\Delta A_{n}(\lambda)\right] & \text { S11d }
\end{array}
$$

In this section we extract the spectrum corresponding to the hot exciton states, $\Delta A_{n}^{h}(\lambda)$. We use Equation S11a as the fit equation in the time window extending from the beginning of the experiment to 500 ps, since all cooling processes take place within this time window, and all recombination processes can be described using a single exponential function. To simplify the analysis, we selected the data obtained at a low pump power $(5 \mu \mathrm{W})$. Under these excitation conditions, only two excited state populations are present in the QD ensemble (i.e. 96.4\% of all excited QDs have single excitons, while 3.5\% have biexcitons). As a result, analysis of this data set required two exponentials with time constants $\tau_{1}$ and $\tau_{2}$ to account for the recombination of the single exciton and biexciton states. Only one exponential with a $\tau^{c}$ time constant was necessary to account for cooling of both the hot biexciton and single exciton states. This is expected as it is known that most cooling time constants are in the order of the instrument response ${ }^{10-11}$; therefore, it is hard to distinguish the cooling time constants of the hot biexcitons and hot single exciton states. A set of preexponential coefficients, $S_{1}(\lambda), S_{2}(\lambda)$, and $S_{n}^{h}(\lambda)$, accompanied each exponential employed in the analysis.

To perform the analysis, we selected kinetic traces every $5 \mathrm{~nm}$ in the spectral window extending from $440 \mathrm{~nm}$ to $646 \mathrm{~nm}$. All kinetic traces were simultaneously analyzed using a global fit routine in which the time constants, $\tau^{c}, \tau_{1}$, and $\tau_{2}$, were considered global parameters and the $S_{1}(\lambda), S_{2}(\lambda)$, and $S_{n}^{h}(\lambda)$ preexponential coefficients were allowed to vary. These fits yielded three time constants, $\left(\tau_{1}=1.6 n s, \tau_{2}=\right.$ $10.6 \mathrm{ps}$, and $\tau^{c}=0.3 \mathrm{ps}$ ) and three sets of pre-exponential coefficients $\left(S_{1}(\lambda), S_{2}(\lambda)\right.$, and $S_{n}^{h}(\lambda)$ ), which are shown in Figure S6 a. The resultant $S_{1}(\lambda), S_{2}(\lambda)$, and $S_{n}^{h}(\lambda)$ were employed in the isolation of the spectrum of the hot exciton states, which was performed through the use of Equation S11e.

$$
\sum_{n=1}^{q} \Delta A_{n}^{h}(\lambda)=S_{n}^{h}(\lambda)+\sum_{n=1}^{q} p_{n} \Delta A_{n} \quad \text { S11e }
$$

The resultant spectrum, which only contains the contribution of hot exciton states, is shown in Figure S6 b. This spectrum has a photoinduced absorption feature to the red of the ground state bleach $(\sim 580 \mathrm{~nm})$, which is more pronounced than that found on the spectra corresponding to the cold exciton states. This result is consistent with prior literature reports, which have predicted and observed that the photoinduced absorption feature of CdSe is more pronounced in the spectrum containing the contribution of the hot exciton states 9 . This finding has been explained by considering how the contributions of state filling and exciton-exciton interactions affect the TA spectra. When the excited electron is hot, (i.e. resides above the band edge), it occupies a high energy state in the conduction band. As a result, there is no state filling contribution to the ground state bleach, and the contribution of exciton-exciton interactions is dominant. On the other hand, when the electron has cooled down to the band edge, the state filling contribution affects both the ground state bleach and the photoinduced absorption feature to the red of it. Since the electron fills one of the doubly degenerate $1 \mathrm{~S}_{\mathrm{e}}$ states, any absorption to the state that is filled is blocked. As a result, in this scenario, the absorption intensity to the $1 S_{e}$ drops by a factor of two with respect to that of the spectrum only containing the contribution of hot exciton states (Figure S6 b). We conclude that the analysis routine presented in the manuscript text is also useful for the isolation of the spectrum of the hot exciton states. 

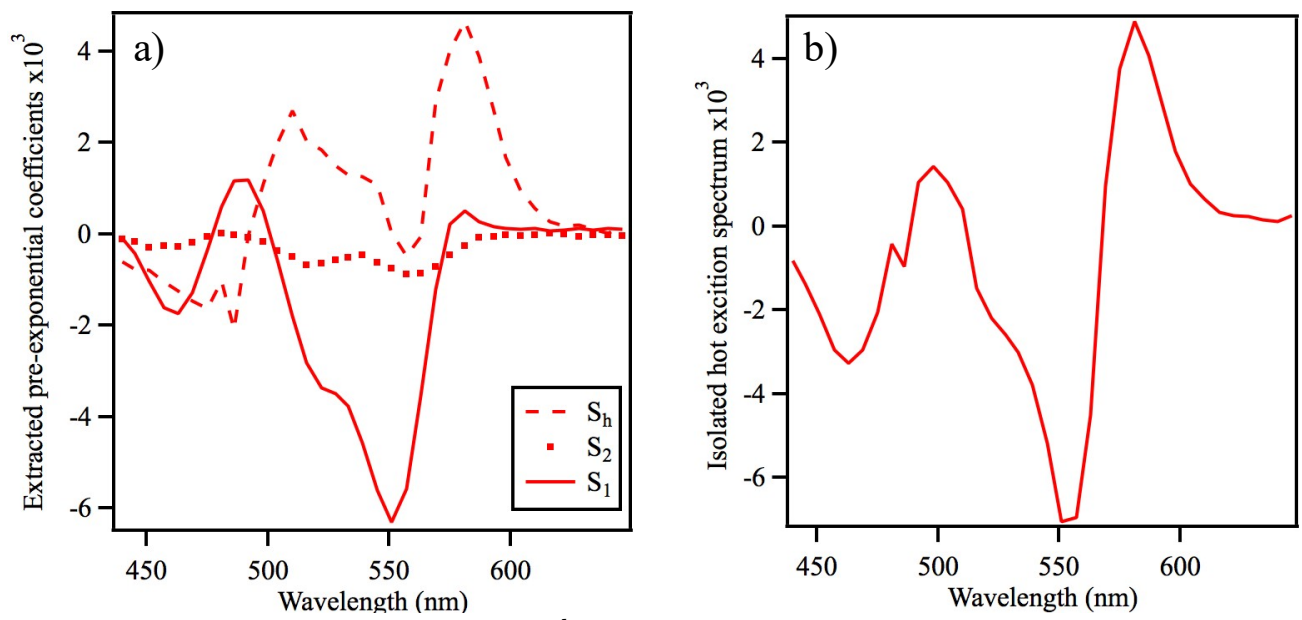

Figure S6: a) Plots of the $S_{1}(\lambda), S_{2}(\lambda)$, and $S_{n}^{h}(\lambda)$ pre-exponential coefficients as a function of wavelength obtained though global fit analysis of the data set excited with a $5 \mu \mathrm{W}$ pump beam. b) Isolated hot exciton spectrum, $\Delta A_{n}^{h}(\lambda)$, extracted through analysis of the same data set.

\section{CITATIONS}

1. Berera, R.; van Grondelle, R.; Kennis, J. T. M. Ultrafast Transient Absorption Spectroscopy: Principles and Application to Photosynthetic Systems. Photosynth. Res. 2009, 101, 105-118.

2. Morris-Cohen, A. J.; Donakowski, M. D.; Knowles, K. E.; Weiss, E. A. The Effect of a Common Purification Procedure on the Chemical Composition of the Surfaces of CdSe Quantum Dots Synthesized with Trioctylphosphine Oxide. J. Phys. Chem. 2010, 114, 897-906.

3. Anderson, N. C.; Hendricks, M. P.; Choi, J. J.; Owen, J. S. Ligand Exchange and the Stoichiometry of Metal Chalcogenide Nanocrystals: Spectroscopic Observation of Facile MetalCarboxylate Displacement and Binding. J. Am. Chem. Soc. 2013, 135, 18536-48.

4. Klimov, V. I.; Mikhailovsky, A. A.; McBranch, D. W.; Leatherdale, C. A.; Bawendi, M. G. Quantization of Multiparticle Auger Rates in Semiconductor Quantum Dots. Science 2000, 287, 1011-1013.

5. Klimov, V. I.; McGuire, J. A.; Schaller, R. D.; Rupasov, V. I. Scaling of Multiexciton Lifetimes in Semiconductor Nanocrystals. Phys. Rev. B 2008, 77, 195324-195335.

6. Ellingson, R. J.; Beard, M. C.; Johnson, J. C.; Yu, P.; Micic, O. I.; Nozik, A. J.; Shabaev, A.; Efros, A. L. Highly Efficient Multiple Exciton Generation in Colloidal PbSe and PbS Quantum Dots. Nano Lett. 2005, 5, 865-71.

7. Berberan-Santos, M. N.; Bodunov, E. N.; Valeur, B. Mathematical Functions for the Analysis of Luminescence Decays with Underlying Distributions: 2. Becquerel (Compressed Hyperbola) and Related Decay Functions. Chem. Phys. 2005, 317, 57-62.

8. Klimov, V.; Hunsche, S.; Kurz, H. Biexciton Effects in Femtosecond Nonlinear Transmission of Semiconductor Quantum Dots. Phys. Rev. B 1994, 50, 8110-8113.

9. Klimov, V. I. Spectral and Dynamical Properties of Multiexcitons in Semiconductor Nanocrystals. Annu. Rev. Phys. Chem. 2007, 58, 635-673.

10. Sewall, S. L.; Cooney, R. R.; Anderson, K. E. H.; Dias, E. A.; Kambhampati, P. State-toState Exciton Dynamics in Semiconductor Quantum Dots. Phys. Rev. B 2006, 74, 235328-235335. 
11. Cooney, R. R.; Sewall, S. L.; Dias, E. A.; Sagar, D. M.; Anderson, K. E. H.; Kambhampati, P. Unified Picture of Electron and Hole Relaxation Pathways in Semiconductor Quantum Dots. Phys. Rev. B 2007, 75, 245311-245324. 